\title{
Contaminación aérea y sus efectos en la salud*
}

\author{
MANUEL OYARZÚN G.**
}

\section{Air pollution an its effects on health}

The term "air pollution" comprises a wide variety of chemical and biological components of the outdoor and indoor atmosphere. Air pollution and its effect on human health is critically reviewed in this article with emphasis in the situation of inhabitants of Chilean cities with critical high levels of atmospheric pollution. Atmospheric contaminants that are hazardous for the human health are "breathable" particulate matter (PM $10 ; P M_{2,5}$ and $\left.P M_{0,1}\right)$ and gaseous chemicals such as nitrogen dioxide, ozone, sulphur dioxide and carbon monoxide. Indoor air contains a variety of noxious compounds derived from multiple sources, being cigarette smoking, heating and cooking appliances, and biological agents and its sub-products the principal ones. Exposure to air pollutants can increase not only morbidity but also mortality rate as well as increasing the number of hospital admissions for patients with respiratory or cardiovascular symptoms. Air pollution is important in determining the quality of life particularly in infants, ageing persons and patients affected by respiratory and cardiovascular diseases. Health professionals should advocate for a cleaner outdoor and indoor atmosphere through the dissemination of our knowledge about the respiratory and non-respiratory effects of air contamination.

Key words: Urban air pollution, indoor air pollution, air quality Chilean standards, respiratory system, cardiovascular, airborne particulate matter.

\section{Resumen}

El término "contaminación del aire” incluye una amplia variedad de componentes químicos y biológicos de la atmósfera intra y extradomiciliaria. Este artículo intenta realizar una revisión crítica de los efectos de la contaminación intra y extradomiciliaria sobre la salud humana, poniendo especial énfasis en la situación de los habitantes de ciudades chilenas con niveles críticamente altos de contaminación atmosférica. Los contaminantes atmosféricos riesgosos para la salud humana son el material particulado inhalable $\left(P M_{10} ; P_{2,5}\right.$ y $\left.P M_{0,1}\right)$ y compuestos químicos gaseosos tales como dióxido de nitrógeno, ozono, dióxido de azufre y monóxido de carbono. El aire intradomiciliario contiene una variedad de compuestos nocivos que derivan de múltiples fuentes. Las más importantes son el humo de cigarrillo, artefactos de calefacción y para cocción de alimentos y los agentes biológicos y sus sub-productos. La exposición a contaminantes del aire no solo puede aumentar la tasa de morbilidad sino la tasa de mortalidad como también puede aumentar el número de ingresos hospitalarios de pacientes con síntomas respiratorios y cardiovasculares. La contaminación del aire es importante en la determinación de la calidad de vida de niños menores, ancianos y en pacientes con enfermedades respiratorias y cardiovasculares. Los profesionales de la salud deberían abogar por una atmósfera intra y extradomiciliaria más limpia a través de la difusión del conocimiento que disponemos sobre los efectos respiratorios y no respiratorios de la contaminación del aire.

Palabras clave: contaminación del aire urbano; contaminación del aire intradomiciliario; normas chilenas de calidad del aire; sistema respiratorio; cardiovascular; material particulado del aire.

* Artículo basado en una conferencia pronunciada en el $1^{\text {er }}$ Congreso de Atención Primaria en Salud: "Mejorando la red asistencial con un enfoque multidisciplinario". Facultad de Ciencias de la Salud, Universidad Católica del Maule. Talca 29.08.2008.

** Director del Programa Disciplinario de Fisiopatología, Instituto de Ciencias Biomédicas, ICBM y Director del Centro de Investigaciones del Medio Ambiente y Biomedicina-CIMAB. Facultad de Medicina. Universidad de Chile. 
La contaminación atmosférica se define como la presencia en la atmósfera de elementos contaminantes que alteran su composición y que afectan a cualquier componente del ecosistema. Desde un punto de vista antropocéntrico la contaminación atmosférica se refiere a los contaminantes que afectan la salud o el bienestar humano ${ }^{1}$.

Según su origen los contaminantes se clasifican en antropogénicos, derivados de la actividad humana, o naturales, resultantes de procesos de la naturaleza, por ejemplo erupciones volcánicas o polen en suspensión ${ }^{1}$.

Según su estado físico son gases como los óxidos de azufre (SOx), de nitrógeno (NOx), el monóxido de carbono (CO), los hidrocarburos y el ozono $\left(\mathrm{O}_{3}\right)$ o partículas como polvo y aerosoles. Se consideran primarios cuando están presentes tal como fueron emitidos y secundarios, cuando se forman a partir de los primarios por una reacción química como es el caso del $\mathrm{O}_{3}$ y de los ácidos sulfúrico y nítrico ${ }^{1}$.

Según su tamaño, las partículas se depositan cerca o a cierta distancia de la fuente de emisión. Si son muy pequeñas pueden mantenerse suspendidas y ser transportadas a grandes distancias. Dentro de las partículas suspendidas se denomina "respirables" a las de un diámetro menor o igual a $10 \mu \mathrm{m}\left(\mathrm{PM}_{10}\right)$ por su capacidad de introducirse en las vías respiratorias. Cuanto más pequeñas son las partículas mayor es su ca- pacidad de penetración en el árbol respiratorio. Las partículas finas cuyo diámetro aerodinámico es $\leq 2,5 \mu \mathrm{m}$ alcanzan fácilmente los bronquíolos terminales y los alvéolos, desde donde pueden ser fagocitadas por los macrófagos alveolares y atravesar la barrera alvéolo-capilar para ser transportadas hacia otros órganos por la circulación sanguínea ${ }^{1}$.

Más recientemente se han descrito las partículas "ultrafinas" cuyo diámetro es aún menor ( $\leq$ $0,1 \mu \mathrm{m})$ y pueden pasar directamente desde los alvéolos al torrente circulatorio. Las partículas pueden contener compuestos orgánicos como los hidrocarburos aromáticos policíclicos e/o inorgánicos como sales y metales ${ }^{1}$.

Se han establecido "normas de calidad del aire", que es el nivel de contaminantes aéreos sobre el cual se espera la aparición de efectos indeseables. Las normas primarias son niveles que protegen la salud de la población con cierto margen de seguridad. Estas normas primarias de calidad del aire (Tabla 1) son actualizadas periódicamente ${ }^{2}$. Actualmente Chile está en el proceso de establecer una norma para $\mathrm{PM}_{2,5}$.

Si bien en Chile la situación de la Región Metropolitana representa el caso más emblemático y notorio de contaminación atmosférica ${ }^{1}$, otras ciudades también presentan altos niveles de contaminación del aire. Se han detectado niveles altos en ciudades cercanas a complejos mineros

Tabla 1. Normas nacionales primarias de calidad del aire*

\begin{tabular}{|c|c|c|c|}
\hline Contaminante & Norma (media) & Unidad & Período de evaluación \\
\hline Partículas $\leq 10 \mu \mathrm{m}\left(\mathrm{PM}_{10}\right)$ & $\begin{array}{r}150 \\
50\end{array}$ & $\begin{array}{l}\mu \mathrm{g} / \mathrm{m}^{3} \mathrm{~N}^{* *} \\
\mu \mathrm{g} / \mathrm{m}^{3} \mathrm{~N}\end{array}$ & $\begin{array}{l}\text { Diario } \\
\text { Anual }\end{array}$ \\
\hline Dióxido de azufre $\left(\mathrm{SO}_{2}\right)$ & $\begin{array}{r}80 \\
250\end{array}$ & $\begin{array}{l}\mu \mathrm{g} / \mathrm{m}^{3} \mathrm{~N} \\
\mu \mathrm{g} / \mathrm{m}^{3} \mathrm{~N}\end{array}$ & $\begin{array}{l}\text { Anual } \\
\text { Diario }\end{array}$ \\
\hline Ozono $\left(\mathrm{O}_{3}\right)$ & $\begin{array}{r}120 \\
60\end{array}$ & $\begin{array}{l}\mu \mathrm{g} / \mathrm{m}^{3} \mathrm{~N} \\
\mathrm{ppb}\end{array}$ & 8 horas \\
\hline Monóxido de carbono (CO) & $\begin{array}{r}30 \\
26 \\
10 \\
9\end{array}$ & $\begin{array}{l}\mathrm{mg} / \mathrm{m}^{3} \mathrm{~N} \\
\mathrm{ppm} \\
\mathrm{mg} / \mathrm{m}^{3} \mathrm{~N} \\
\mathrm{ppm}\end{array}$ & $\begin{array}{l}1 \text { hora } \\
8 \text { horas }\end{array}$ \\
\hline Dióxido de nitrógeno $\left(\mathrm{NO}_{2}\right)$ & $\begin{array}{l}100 \\
400\end{array}$ & $\begin{array}{l}\mu \mathrm{g} / \mathrm{m}^{3} \mathrm{~N} \\
\mu \mathrm{g} / \mathrm{m}^{3} \mathrm{~N}\end{array}$ & $\begin{array}{l}\text { Anual } \\
1 \text { hora }\end{array}$ \\
\hline Plomo $(\mathrm{Pb})$ & 0,5 & $\mu \mathrm{g} / \mathrm{m}^{3} \mathrm{~N}$ & Anual \\
\hline
\end{tabular}

*Decretos Supremos: 59/1998 y 136/2000. Las medias aritméticas no deben superarse en 3 años sucesivos, excepto en el caso del $\mathrm{Pb}$, que no debe superarse en 2 años sucesivos. ${ }^{* *} \mathrm{~m}^{3} \mathrm{~N}=$ metro cúbico normal (a $25^{\circ} \mathrm{C}$ y $760 \mathrm{mmHg}$ ); $\mathrm{ppm}=$ partes por millón $\left(1 \times 10^{6}\right) ; \mathrm{ppb}=$ partes por billón USA $\left(1 \times 10^{9}\right)$. Equivalencia entre $\mathrm{ppm}$ y $\mu \mathrm{g} / \mathrm{m}^{3} \mathrm{~N}: \mathrm{ppm}=$ $\left[24,45 \cdot\left(\mathrm{mg} / \mathrm{m}^{3}\right)\right] /$ peso molecular en gramos. 
como Copiapó y Rancagua o industriales como Puchuncaví $^{3}$ y también en ciudades del sur de Chile que usan leña como fuente energética en invierno (Osorno, Temuco 4 y Coyhaique). En 2004, Temuco fue declarada zona saturada para $\mathrm{PM}_{10}$, los niveles más elevados de $\mathrm{PM}_{10}$ se detectan en invierno y en horario vespertino. En la Región del Maule, Talca registra altos niveles de contaminación atmosférica, especialmente en el sector La Florida (Figura 1). Las concentraciones atmosféricas de material particulado fino, medidas en este sector de Talca, indican que durante los meses de mayo a agosto se estarían superando las guías de calidad del aire de la Organización Mundial de la Salud, por lo que la población expuesta estaría con niveles de riesgo inaceptables.

\section{Efectos en la salud $^{5,6}$}

Los efectos de los contaminantes sobre la salud se han estudiado a través de diversos modelos experimentales (exposición a contaminantes de células, tejidos, animales y voluntarios) y epidemiológicos (episodios de contaminación, comparación de poblaciones expuestas versus no expuestas, sanos versus enfermos y metaanálisis). Considerados separadamente, cada uno de estos tipos de estudios tiene fortalezas y debilidades. Por lo tanto, es el conjunto de resultados obtenidos aplicando estos diferentes diseños en el estudio de los contaminantes, lo que les confiere mayor valor a los efectos encontrados.
El espectro de la respuesta biológica a los contaminantes es muy amplio y puede ser representado como una pirámide, cuya base está constituida por toda la población expuesta, luego le sigue un estrato de la población que tiene acúmulos de contaminantes en su organismo, un tercer nivel está representado por un segmento de la población que presenta cambios bioquímicos de significado incierto; un cuarto nivel es el segmento que presenta cambios funcionales que pueden ser precursores de enfermedad. Hasta aquí llega la etapa subclínica de la exposición. El penúltimo nivel de la pirámide de la población expuesta está constituido por quienes presentan morbilidad y la cúspide de la pirámide está constituida por quienes mueren a consecuencia de los contaminantes.

\section{Efectos de los contaminantes sobre el sistema respiratorio ${ }^{1,5,6}$}

Los efectos adversos dependen por una parte, de la concentración y la duración de la exposición y por otra, de la susceptibilidad de las personas expuestas. La dosis efectivamente recibida es dependiente de la ventilación minuto, según la siguiente fórmula:

\section{Dosis efectiva $=[$ Concentración $] \cdot[$ Tiempo de exposición] · [Ventilación minuto]}

Entre los factores que aumentan la ventilación,

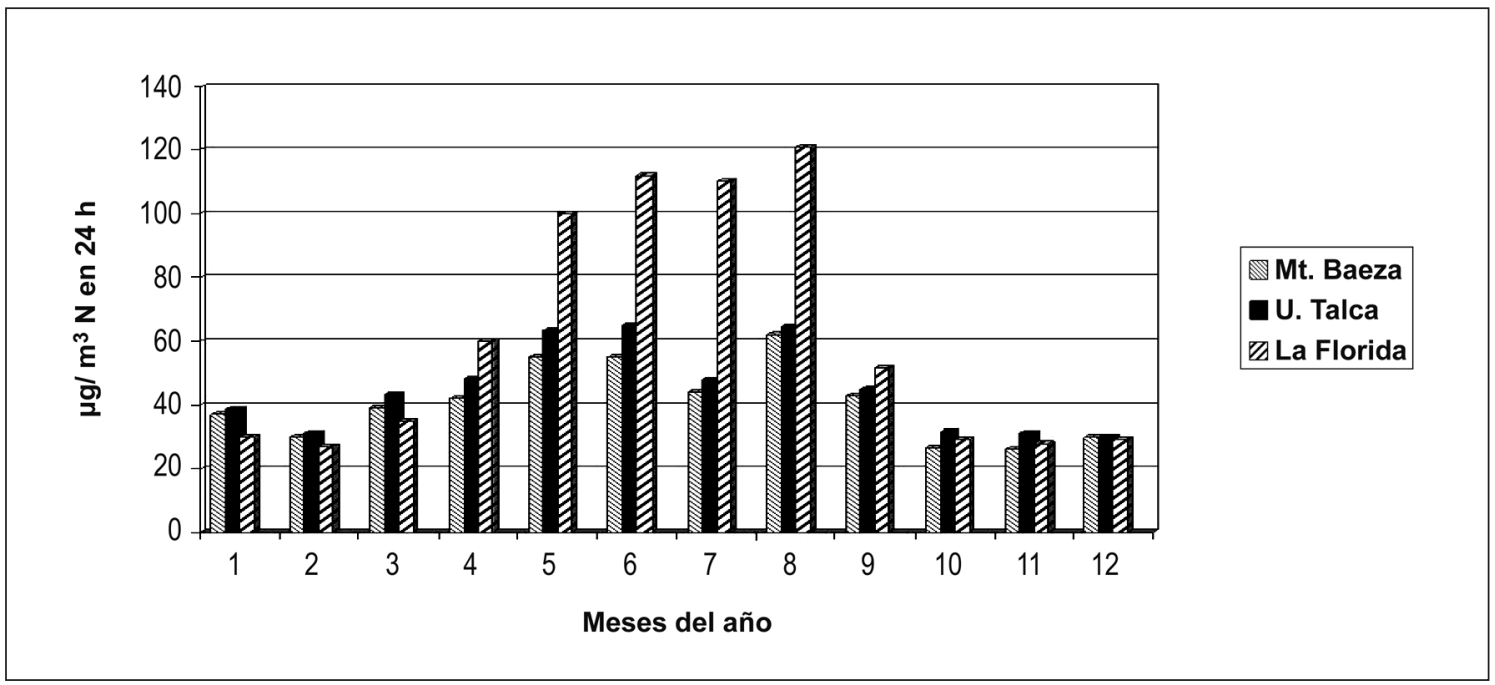

Figura 1. Promedios mensuales de $\mathrm{PM}_{10}$ registrados en Talca durante 2007. Las columnas representan los niveles de $\mathrm{PM}_{10}$ en $\mu \mathrm{g} / \mathrm{m}^{3}$ normal en $24 \mathrm{~h}$, medidos en las estaciones situadas en los sectores de Monte Baeza, Universidad de Talca y La Florida. El gráfico fue elaborado con datos proporcionados por la Sra. Elisa Muñoz, Programa Calidad del Aire, SEREMI, VII Región. 
Tabla 2. Factores que explican la mayor susceptibilidad del sistema respiratorio a los contaminantes atmosféricos en la infancia y en la senectud ${ }^{5,7}$

\begin{tabular}{|ll|}
\hline Infancia & Senectud \\
$\begin{array}{l}\text { Menor efectividad de la tos por menor desarrollo de la } \\
\text { musculatura respiratoria }\end{array}$ & $\begin{array}{l}\text { Disminución de la fuerza de los músculos inspiratorios } \\
\text { y menor efectividad de la tos }\end{array}$ \\
$\begin{array}{l}\text { Mayor ventilación por mayor frecuencia respiratoria } \\
\text { en reposo, aumenta la dosis efectiva de contaminantes }\end{array}$ & Rigidez torácica \\
$\begin{array}{l}\text { Ausencia de ventilación colateral agrava la obstrucción } \\
\text { de vías aéreas periféricas ( }<2 \text { mm de diámetro) }\end{array}$ & $\begin{array}{l}\text { Disminución de la respuesta ventilatoria a la hipoxia y } \\
\text { a la hipercapnia }\end{array}$ \\
$\begin{array}{l}\text { Mayor resistencia de las vías aéreas periféricas genera } \\
\text { el } 50 \% \text { de la resistencia total al flujo aéreo (en el adulto } \\
\text { es }<20 \%)\end{array}$ & Disminución de la percepción de obstrucción bronquial \\
$\begin{array}{l}\text { Menor volumen pulmonar y menor superficie alveolar } \\
\text { Mecanismos defensivos no plenamente desarrollados y } \\
\text { mayor dificultad en la eliminación de partículas desde } \\
\text { las vías aéreas }\end{array}$ & $\begin{array}{l}\text { Disminución de la superficie alveolar por "enfisema } \\
\text { senil" }\end{array}$ \\
$\begin{array}{l}\text { Mayor exposición a contaminantes atmosféricos, por- } \\
\text { que los niños pasan mayor parte de su tiempo al aire }\end{array}$ & \\
\hline libre
\end{tabular}

elevando la carga de contaminantes que recibe el pulmón, destaca el aumento de la temperatura y humedad que dificultan la termólisis, el aumento de la altitud, que lleva a la disminución de la presión inspirada de $\mathrm{O}_{2}$ y por ende a hipoxemia hipobárica, el aumento de la progesterona (en el embarazo), estados febriles y el ejercicio físico. Todas ellas son condiciones que provocan un aumento de la frecuencia respiratoria y del volumen corriente que son los factores determinantes de la ventilación minuto.

Los niños y senescentes son especialmente susceptibles a los efectos de los contaminantes (Tabla 2). Por otra parte, la presencia de enfermedades respiratorias y cardiovasculares también aumenta la vulnerabilidad a los contaminantes aéreos, ya que estas condiciones se acompañan de disnea, aumento de la ventilación minuto y dificultad de depuración de las vías respiratorias por edema, inflamación, limitación del flujo aéreo o por disminución de la capacidad de movilización de volúmenes pulmonares.

Los principales contaminantes aéreos se han asociado a efectos específicos sobre el sistema respiratorio (Tabla 3). Sin embargo, las personas están habitualmente expuestas a una mezcla de ellos, lo que puede potenciar los efectos atribuidos a cada uno. Es así como se ha descrito poten- ciación de efectos entre $\mathrm{PM}_{10}$ y SOx y entre $\mathrm{SOx}$ y $\mathrm{O}_{3}$ y más recientemente entre $\mathrm{PM}_{10}$ y ozono.

Los contaminantes atmosféricos también contribuyen en la disminución de la función pulmonar y al aumento de la reactividad bronquial, disminuir la tolerancia al ejercicio y a aumentar el riesgo de bronquitis obstructiva crónica, enfisema pulmonar, exacerbación del asma bronquial y cáncer pulmonar, entre otros efectos. En Chile, desde 1980 han proliferado los estudios que demuestran efectos de la contaminación atmosférica, especialmente de las partículas, sobre la mortalidad diaria, síntomas y consultas respiratorias. Estos estudios realizados primero en Santiago y luego en Temuco ${ }^{4}$, han confirmado los resultados comunicados en publicaciones internacionales que han establecido que por cada $50 \mu \mathrm{g} /$ $\mathrm{m}^{3}$ de elevación de los niveles de $\mathrm{PM}_{10}$ en $24 \mathrm{~h}$ se produce en promedio un aumento de alrededor del 3\% de la mortalidad general. También estos estudios han detectado que el aumento de $\mathrm{PM}_{10}$ se asocia a aumento de la mortalidad respiratoria y cardiovascular.

En relación al $\mathrm{PM}_{2,5}$ estudios nacionales han encontrado que un aumento de la concentración de $\mathrm{PM}_{2,5}$ por encima de $70 \mu \mathrm{g} / \mathrm{m}^{3}$ propicia el aumento de las consultas por neumonías infantiles $^{8}$. Se ha propuesto un nivel de $63 \mu \mathrm{g} / \mathrm{m}^{3} \mathrm{~N}$ 
Tabla 3. Efectos adversos de los contaminantes aéreos sobre el sistema respiratorio ${ }^{5,6}$

\begin{tabular}{|c|c|c|}
\hline Contaminante & Efecto a corto plazo & Efecto a largo plazo \\
\hline $\begin{array}{l}\text { Material particulado } \\
\text { "respirable" } \\
\left(\mathrm{PM}_{10}\right) \text { y fino }\left(\mathrm{PM}_{2,5}\right)\end{array}$ & $\begin{array}{l}\text { Aumento de morbimortalidad respiratoria } \\
\text { Disminución en la función pulmonar } \\
\text { Interferencia en mecanismos de } \\
\text { defensa pulmonar: fagocitosis y depuración } \\
\text { mucociliar } \\
\text { Síndrome bronquial obstructivo }\end{array}$ & $\begin{array}{l}\text { Menor desarrollo de la } \\
\text { estructura y función del } \\
\text { sistema respiratorio } \\
\text { Mayor riesgo de cáncer en } \\
\text { la edad adulta (HAPs) }\end{array}$ \\
\hline $\begin{array}{l}\text { Particulado ultrafino } \\
\left(\mathrm{PM}_{0,1}\right)\end{array}$ & $\begin{array}{l}\text { Mayor respuesta inflamatoria. (comparado con } \mathrm{PM}_{10} \\
\text { y } \mathrm{PM}_{2,5} \text { ) } \\
\text { Pasaje rápido a la circulación y a otros órganos }\end{array}$ & \\
\hline Ozono $\left(\mathrm{O}_{3}\right)$ & $\begin{array}{l}\text { Disminución de frecuencia respiratoria y disminución } \\
\text { de CVF y VEF } \\
\text { Alveolitis neutrofílica, aumento de permeabilidad e } \\
\text { hiperreactividad bronquial } \\
\text { Alteración del epitelio alveolar (células tipo II) }\end{array}$ & $\begin{array}{l}\text { Daño de células epiteliales, } \\
\text { "bronquiolización" alveolar } \\
\text { Disminución del desarrollo } \\
\text { de CVF y } \mathrm{VEF}_{1}\end{array}$ \\
\hline $\begin{array}{l}\text { Dióxido de azufre } \\
\left(\mathrm{SO}_{2}\right)\end{array}$ & $\begin{array}{l}\text { Obstrucción bronquial } \\
\text { Hipersecreción bronquial }\end{array}$ & Bronquitis crónica \\
\hline $\begin{array}{l}\text { Dióxido de nitrógeno } \\
\left(\mathrm{NO}_{3}\right)\end{array}$ & $\begin{array}{l}\text { Hiperreactividad bronquial } \\
\text { Aumento de síntomas respiratorios y exacerbaciones } \\
\text { de asma } \\
\text { Aumenta la respuesta a la provocación con alergenos } \\
\text { Disminución de la actividad mucociliar }\end{array}$ & $\begin{array}{l}\text { Posible decremento del } \\
\text { desarrollo pulmonar }\end{array}$ \\
\hline $\begin{array}{l}\text { Monóxido de carbono } \\
\text { (CO) }\end{array}$ & Disminución en la capacidad de ejercicio & \\
\hline Plomo $(\mathrm{Pb})$ & Alteración del epitelio bronquiolar (células de Clara) & \\
\hline
\end{tabular}

CVF: Capacidad vital forzada; $\mathrm{VEF}_{1}$ : Volumen espiratorio forzado en el primer segundo. HAPs: hidrocarburos aromáticos policíclicos.

como norma diaria de $\mathrm{PM}_{2,5}$ para el año 2012 (Resolución Exenta $\mathrm{N}^{\circ} 4624$ de CONAMA del 10.08.2009). Por otra parte, un aumento de 10 $\mu \mathrm{g} / \mathrm{m}^{3}$ en el promedio diario de $\mathrm{PM}_{2,5}$ se asoció a un aumento de $5 \%$ del riesgo de síndrome bronquial obstructivo con un día de rezago en un seguimiento de 504 lactantes menores de 1 año usuarios de consultorios del área Sur-Oriente de Santiago. La asociación entre $\mathrm{PM}_{2,5}$ y síndrome bronquial obstructivo se mantuvo hasta con 9 días de rezago y fue más evidente en lactantes con historia de asma familiar que en lactantes sin este antecedente ${ }^{9,10}$.

Los efectos agudos de la exposición a $\mathrm{PM}_{10}$ y dióxido de azufre sobre la salud respiratoria fueron evaluados durante 66 días en 114 niños de 6 a 12 años que habitaban el área de influencia del complejo industrial Puchuncaví-Ventanas en la Región de Valparaíso, encontrándose que la exposición a estos contaminantes redujo el flujo espiratorio cúspide (PEF) y aumentó significati- vamente la ocurrencia de tos y expectoración y el uso de broncodilatadores en los niños inicialmente sintomáticos respiratorios. En los niños asintomáticos, la exposición redujo el PEF y aumentó la ocurrencia de sibilancias. Concluyéndose que la salud respiratoria de estos niños estaba siendo afectada por estos contaminantes siendo urgente y necesario disminuir los niveles de contaminación atmosférica en esa zona ${ }^{3}$.

\section{Efectos de los contaminantes sobre otros órganos y sistemas}

Estos efectos son múltiples y pueden afectar a los diferentes órganos y sistemas con diversos grados de intensidad (Tabla 4). En las últimas décadas se ha puesto énfasis en los efectos cardiovasculares. La morbimortalidad por enfermedades cardiovasculares aumenta con la contaminación atmosférica especialmente de $\mathrm{PM}_{10}$ y $\mathrm{CO}$, 
Tabla 4. Efectos no respiratorios de los contaminantes atmosféricos

\begin{tabular}{|c|c|c|}
\hline Órganos / Sistemas & Contaminantes & Efectos \\
\hline \multirow[t]{4}{*}{ Cardiovascular } & Material particulado & $\begin{array}{l}\text { Disminución de la variabilidad en la frecuencia } \\
\text { cardíaca ante el estrés }\end{array}$ \\
\hline & Monóxido de carbono & Interfiere el transporte de $\mathrm{O}_{2}$ por la hemoglobina \\
\hline & Plomo / Vanadio & $\begin{array}{l}\text { Mayor frecuencia de hipertensión arterial en pobla- } \\
\text { ción adulta }\end{array}$ \\
\hline & Ozono $\left(\mathrm{O}_{3}\right)$ & $\begin{array}{l}\text { Comunicación interventricular (administración } \\
\text { prenatal en ratas) }\end{array}$ \\
\hline Unidad materno-fetal & $\begin{array}{l}\text { Monóxido de carbono y } \mathrm{PM}_{2,5} \\
\text { (hidrocarburos aromáticos } \\
\text { policíclicos: HAP) }\end{array}$ & $\begin{array}{l}\text { Bajo peso de nacimiento } \\
\text { Baja talla al nacer }\end{array}$ \\
\hline \multirow[t]{3}{*}{$\begin{array}{l}\text { Sistema nervioso } \\
\text { central y autonómico }\end{array}$} & Monóxido de carbono & $\begin{array}{l}\text { Cefalea, irritabilidad, disminución de percepción } \\
\text { auditiva y visual. Compromiso progresivo y letal de } \\
\text { conciencia en concentraciones altas }\end{array}$ \\
\hline & Plomo & $\begin{array}{l}\text { Hiperquinesia, trastornos del aprendizaje; encefalo- } \\
\text { patía; cólicos intestinales }\end{array}$ \\
\hline & Ozono $\left(\mathrm{O}_{3}\right)$ & $\begin{array}{l}\text { Daño cerebeloso en células de Purkinje (administra- } \\
\text { do prenatalmente en ratas) }\end{array}$ \\
\hline Renal & $\begin{array}{l}\text { Cadmio y Vanadio } \\
\text { Plomo }\end{array}$ & $\begin{array}{l}\text { Toxicidad renal } \\
\text { Tubulopatía }\end{array}$ \\
\hline Hematopoyético & Plomo & Anemia \\
\hline Óseo & Plomo & $\begin{array}{l}\text { Reemplazo del } \mathrm{Ca}^{+2} \text { en los huesos produciendo } \\
\text { descalcificación }\end{array}$ \\
\hline
\end{tabular}

especialmente por arterioesclerosis, arritmias e insuficiencia coronaria en adultos ${ }^{11}$.

Otro hecho relevante es la presencia de hidrocarburos aromáticos policíclicos (HAPs), como componentes del material particulado. Estos compuestos se generan por la combustión incompleta de material orgánico (petróleo, gasolina, leña, carbón y biomasa en general). En la fracción orgánica del material particulado de ciudades con altos niveles de contaminación atmosférica se han identificado numerosas especies de HAPs; seis de ellos han sido catalogados como cancerígenos por la International Agency of Research on Cancer, siendo el benzo $\alpha$-pireno el HAP más cancerígeno presente en el humo del cigarrillo y en el smog de ciudades con alta contaminación incluyendo a Santiago. Estos HAPs pueden reaccionar con $\mathrm{NO}_{2}$ generando nitroarenos que tienen gran actividad mutagénica. Aunque en Santiago aún no existen estudios epidemiológicos acerca de la influencia de los HAPs atmosféricos en la génesis de cáncer pul- monar o en cánceres de otros órganos o sistemas, las concentraciones aéreas de estos compuestos sugieren que podrían tener algún papel en la incidencia de esta enfermedad. Un estudio prospectivo de mortalidad sobre un total de 1,2 millones de estadounidenses seguidos durante 16 años (198298) comunicó que un aumento de $\mathrm{PM}_{2,5}$ en $10 \mu \mathrm{g} /$ $\mathrm{m}^{3}$ se asoció a aumento en el riesgo de mortalidad por cáncer pulmonar de aproximadamente $8 \%$, de $4 \%$ de mortalidad global y de $6 \%$ de mortalidad cardiopulmonar ${ }^{12}$.

En resumen, la evidencia acumulada hasta ahora indica que los contaminantes atmosféricos son responsables de contribuir al aumento de la mortalidad general ${ }^{13}$, de la mortalidad infantil, de la mortalidad de mayores de 65 años y de las hospitalizaciones por enfermedades respiratorias y cardíacas. Todos ellos son efectos indeseables para la salud de la población expuesta, de allí la importancia de lograr controlarla y eventualmente abatirla $^{14,15}$. 


\section{Contaminación intradomiciliaria ${ }^{16}$}

Los compuestos que forman parte de la contaminación atmosférica pueden también contaminar el aire ambiental intradomiciliario. Sin embargo, el aire atmosférico se difunde y distribuye en forma más homogénea afectando en forma más o menos similar a los miembros de una comunidad, no ocurre lo mismo con el aire intradomiciliario, cuya calidad es muy dependiente del nivel socioeconómico de los moradores.

Las principales fuentes de contaminación del aire intradomiciliario son el tabaquismo, la quema inadecuada de combustibles, el hacinamiento, y la convivencia y cohabitación con animales domésticos.

Las personas más afectadas por la contaminación intradomiciliaria son quienes pasan la mayor parte de su tiempo en espacios cerrados, es decir, los niños menores de 2 años de edad, los ancianos y los enfermos ${ }^{16}$.

La dilución de contaminantes en espacios cerrados depende del volumen del aire habitacional, el cual es función del tamaño y diseño de la construcción. Por otra parte, su eliminación depende del grado de aislamiento y vinculación con el exterior y de la existencia de sistemas de filtros.

La calidad del aire en espacios cerrados es multifactorial dependiendo especialmente de los hábitos de los moradores, de su nivel socioeconómico, del intercambio de aire con el exterior y de la remoción de los contaminantes (Tabla 5).

En términos de emisión (Tabla 6), los contaminantes pueden diferenciarse en productos de la combustión, agentes biológicos (microorganismos y alergenos); y misceláneos: radón y compuestos orgánicos volátiles (COVs) como benceno, tolueno, formaldehídos y diluyentes como el tetracloroetileno. Aunque estos últimos tienen un potencial tóxico importante, son los productos de la combustión los más importantes en términos poblacionales. Por su parte, la exposición intradomiciliaria a radón está asociada a cáncer pulmonar ${ }^{17}$.

\section{Efectos en la salud}

Humo de tabaco ambiental. Es el principal componente de la contaminación intradomiciliaria. El tabaquismo en Chile tiene alta prevalencia en jóvenes escolares de ambos sexos. Este hecho puede determinar un futuro aumento de la exposición en niños pequeños, ya que las madres constituyen su fuente primaria de exposición. En el área Sur-Oriente de Santiago hemos detectado que los niveles de cotinina (biomarcador de la exposición a nicotina) aumentan $40 \%$ en lactantes
Tabla 5. Factores determinantes de la calidad del aire intradomiciliario

Hábitos de los moradores

- Tabaquismo

Nivel socioeconómico

- Hacinamiento

- Uso de combustibles

Intercambio de aire con el ambiente externo

- Puertas y ventanas, sistemas de aislación y ventilación

Remoción de contaminantes

- Superficie del suelo

- Volumen de aire en habitaciones

- Diseño de la construcción

- Eficiencia de la ventilación

- Sistema de aire acondicionado

Tabla 6. Contaminantes intradomiciliarios según origen

\begin{tabular}{|ll|}
\hline $\begin{array}{l}\text { Derivados de la } \\
\text { combustión }\end{array}$ & $\begin{array}{l}\text { Agentes biológicos y } \\
\text { sub-productos }\end{array}$ \\
\hline Humo de tabaco & $\begin{array}{l}\text { Virus, bacterias, hongos y } \\
\text { protozoos }\end{array}$ \\
\hline Humo de leña & Mascotas: perros y gatos \\
\hline Dióxido de nitrógeno & Insectos, ácaros y pólenes \\
\hline Monóxido de carbono & Misceláneos \\
\hline Dióxido de carbono & $\begin{array}{l}\text { Compuestos orgánicos } \\
\text { volátiles (COV) y radón }\end{array}$ \\
\hline
\end{tabular}

cuyo padre es fumador alcanzando un $200 \%$ de aumento respecto a hijos de no fumadores, si la madre es la fumadora ${ }^{18}$.

Las consecuencias de la exposición al humo de tabaco ambiental son múltiples tanto en niños como en adultos (Tabla 7). En los niños aumenta la ocurrencia de infecciones respiratorias bajas y la frecuencia de síntomas respiratorios; reduce el crecimiento pulmonar y el nivel esperado de $\mathrm{VEF}_{1}$ y $\mathrm{FEF}_{25-75}$ y aumenta el riesgo de desarrollar otitis media y de muerte súbita. En los adultos, sus consecuencias serían similares a los de la exposición activa: aumento del riesgo de cáncer pulmonar e isquemia coronaria en la edad adulta $^{19}$. 
Tabla 7. Efectos de la exposición al humo de tabaco ambiental sobre la salud ${ }^{19}$

\begin{tabular}{|ll|}
\hline Cardiovasculares & $\begin{array}{l}\text { Aumento en } 20 \text { a } 50 \% \text { el riesgo de morbilidad por cardiopatías } \\
\text { Aumento morbilidad por cardiopatía coronaria aguda (25 a 35\%) } \\
\text { Riesgo }=1 / 3 \text { de los fumadores activos }\end{array}$ \\
& $\begin{array}{l}\text { Aumento síntomas respiratorios en niños } \\
\text { Respiratorias }\end{array}$ \\
& $\begin{array}{l}\text { Aumento infecciones respiratorias bajas en niños (OR:1,41 en lactantes) } \\
\text { Aumento de exacerbación de asma bronquial en niños y adultos }\end{array}$ \\
Salud reproductiva & Bajo peso de nacimiento (RN) \\
& $\begin{array}{l}\text { Aumento de RN pequeños para la edad gestacional } \\
\text { Desórdenes menstruales, adelantamiento de menopausia en 2 años }\end{array}$ \\
Síndrome de muerte súbita infantil & Aumento del riesgo en 2,5 veces \\
Cáncer & Aumento en 20 a 30\% del riesgo de cáncer pulmonar (EPA y Reino Unido) \\
& Aumento en cáncer mamario en premenopáusicas (OR: 1,40$)$ \\
& Aumento en cáncer de senos nasales en mujeres (OR: 1,21$)$
\end{tabular}

RN: recién nacido; OR: odds ratio (razón de disparidad). EPA: Agencia de Protección del Ambiente, USA.

\section{Humo de la combustión de leña}

La combustión de biomasa es muy incompleta, generando una gran cantidad de compuestos orgánicos parcialmente oxidados. Las partículas generadas son ultrafinas $(<0,1 \mu \mathrm{m})$, lo que les permite traspasar fácilmente la barrera mucociliar, depositarse en bronquíolos y alvéolos y pasar directamente a la sangre. En voluntarios se ha encontrado evidencia que se produce una respuesta inflamatoria sistémica. Por otra parte, un gran número de estudios epidemiológicos en países desarrollados y en desarrollo han detectado en niños efectos mayores a los atribuidos al material particulado atmosférico. Es preocupante que se haya encontrado que in vitro los hidrocarburos aromáticos policíclicos del humo de leña pueden ser mutagénicos.

Dióxido de nitrógeno ( $\left.\mathrm{NO}_{2}\right)$. Su principal fuente intradomiciliaria es la combustión de artefactos domésticos a gas propano, natural o a kerosene (parafina). En general, las concentraciones de $\mathrm{NO}_{2}$ que se registran habitualmente al interior de los domicilios en países desarrollados, no han sido implicadas en efectos adversos para la salud humana.

Monóxido de carbono (CO). Sus fuentes intradomiciliarias son el humo del tabaco, los calefonts a gas mal ventilados (principal causa de intoxicación letal por $\mathrm{CO}$ en Chile), estufas y llamas de "pilotos" a gas, estufas y chimeneas a leña, a kerosene, motores a gasolina, linternas y cocinillas de excursión, conductos de ventilación mal diseñados y vecindad con garajes y calles con alto flujo de vehículos motorizados. Sus efectos se deben a la hipoxia que se produce porque el $\mathrm{CO}$ desplaza al $\mathrm{O}_{2}$ de la hemoglobina y también del sistema citocromo A mitocondrial produciendo según su concentración sanguínea desde cefalea e irritabilidad a compromiso de conciencia progresiva y muerte por hipoxia.

Dióxido de carbono $\left(\mathrm{CO}_{2}\right)$. El $\mathrm{CO}_{2}$ es un producto del metabolismo animal siendo emitido constantemente por los seres humanos y animales que habitan los espacios cerrados. Otras fuentes del $\mathrm{CO}_{2}$ intradomiciliario son los calefactores a gas o a kerosene y el humo del tabaco.

Una habitación bien ventilada tiene una concentración de $\mathrm{CO}_{2}$ de $1.000 \mathrm{ppm}$ con un aporte de aire fresco de 15 pies cúbicos por persona. El mecanismo de daño del $\mathrm{CO}_{2}$ consiste en generar hipoxemia por disminución de la $\mathrm{PO}_{2}$ en el aire inspirado al aumentar primariamente la $\mathrm{PCO}_{2}$ en el aire inspirado, lo que además produce hipercapnia. Las manifestaciones clínicas son hiperventilación, sudoración, cefalea, aumento de la temperatura cutánea y compromiso gradual de conciencia.

\section{Contaminantes biológicos}

$\mathrm{Su}$ presencia y concentración contribuye a determinar la calidad del aire de los espacios 
cerrados y pueden causar graves consecuencias como la enfermedad de los legionarios (asociada a sistemas de aire acondicionado), infecciones o enfermedades alérgicas.

Los agentes biológicos son habitualmente microscópicos: virus, bacterias, hongos y protozoos. También pueden ser insectos (polillas, pulgas y cucarachas), ácaros (dermatofagoides) y pólenes.

Los contaminantes biológicos pueden afectar las vías respiratorias altas y bajas a través de reacciones inmunológicas o provocando infección. Pueden contaminar el aire intramural por diversos mecanismos entre los que se destacan la diseminación a través de la ventilación, dilución acuosa de moléculas mayores generando bioaerosoles y la invasión de espacios por destrucción del hábitat original. En ambientes húmedos, el crecimiento de hongos y la producción de micotoxinas pueden afectar el sistema respiratorio (asma y eventual hemorragia pulmonar). La contaminación por hongos debería ser controlada especialmente en viviendas sociales afectadas por la humedad.

\section{Contaminantes misceláneos}

Numerosos contaminantes ambientales intradomiciliarios han sido sindicados como desencadenantes de asma (humo de tabaco, hongos, ácaros, cucarachas, caspa de animales y plaguicidas). Más recientemente benceno y 1,3 butadieno y algunos plaguicidas se han relacionado con neoplasias infantiles. Un estudio de la American Academy of Pediatrics ${ }^{20}$ sugiere que un $28 \%$ de las discapacidades del desarrollo en niños puede ser causada por factores ambientales aislados o actuando en conjunto con factores genéticos.

En resumen, la contaminación del aire intradomiciliario o de los espacios cerrados es un importante factor a evaluar para mejorar la calidad de vida en las edades extremas de la vida. La contaminación del aire intradomiciliario es muy dependiente del nivel socioeconómico y de los hábitos de los moradores, por lo que las medidas educativas y de protección social son muy importantes para lograr su control.

\section{Bibliografía}

1.- MORALES R G E. Contaminación atmosférica urbana. Episodios críticos de contaminación ambiental en la ciudad de Santiago. Editorial Universitaria SA, Santiago de Chile, 2006.

2.- MATUS P, LUCERO R. Norma primaria de calidad del aire. Rev Chil Enf Respir 2002; 18: 112-22.
3.- SÁNCHEZ J, ROMIEU I, RUIZ S, PINO P, GUTIÉRREZ M. Efectos agudos de las partículas respirables y del dióxido de azufre sobre la salud respiratoria en niños del área industrial de Puchuncaví, Chile. Rev Panam Salud Pública 1999; 6: 384-91.

4.- SANHUEZA P, VARGAS C, MELLADO P. Impacto de la contaminación del aire por PM10 sobre la mortalidad diaria de Temuco. Rev Med Chile 2006; 34: 754-61.

5.- D'AMATO G, HOLGATE S T. The impact of air pollution on respiratory health. European Respiratory Monograph 2002; 7: 1-282.

6.- AMERICAN THORACIC SOCIETY COMMITTEE OF THE ENVIRONMENTAL AND OCCUPATIONAL HEALTH ASSEMBLY. Health effects of outdoor air pollution. Am J Respir Crit Care Med 1996; 153: 3-50 (Part 1) y 153: 477-98 (Part 2).

7.- OYARZÚN M. Función respiratoria en la senectud. Rev Med Chile 2009; 137: 411-8.

8.- ILABACA M, OLAETA I, CAMPOS E, VILLAIRE J, TÉLLEZ-ROJO M M, ROMIEU I. Association between levels of fine particulate and emergency visits for pneumonia and other respiratory illness among children in Santiago, Chile. J Air Waste Manag Assoc 1999; 48: 174-83.

9.- OYARZÚN M, PINO P, ORTIZ J, OLAETA I. Effect of atmospheric pollution on the respiratory system. Biol Res 1998; 31: 361-66.

10.- PINO P, WALTER T, OYARZÚN M, VILLEGAS R, ROMIEU I. Fine particulate levels and the incidence of wheezing illnesses in the first year of life. Epidemiology 2004; 15: 702-8.

11.- PETERS A, VON KLOT S, HEIER M, TRENTINAGLIA I, HÖRMANN A, WICHMANN H E, et al. Exposure to traffic and the onset of myocardial infarction. N Engl J Med 2004; 351: 1721-30.

12.- POPE C A, BURNETT R T, THUN M J, CALLE E E, KREWSKI D, ITO K, et al. Lung cancer, cardiopulmonary mortality, and long-term exposure to fine particulate air pollution. JAMA 2002; 287: 1132-41.

13.- OSTRO B, SÁNCHEZ J M, ARANDA C, ESKELAND G. Air pollution and mortality. Results from study from Santiago Chile. J Expo Anal Environ Epidemiol 1996; 6: 97-114.

14.- OYARZÚN M. Respirar aire limpio: Un derecho constitucional difícil de alcanzar (Editorial). Rev Chil Enf Respir 1999; 22: 151-3.

15.- OYARZÚN M. Desencuentros entre los conocimientos y las políticas públicas para afrontar la contaminación atmosférica (Editorial). Rev Chil Enf Respir 2006; 22 : 151-3.

16.- SAMET M, MARBUCY M C, SPENGLER M. Health effects and sources of indoor air pollution (Part I). Am Rev Respir Dis 1987; 136: 486-508. Part II Am Rev Respir Dis 1987; 137: 221-42.

17.- FIELD R W, STECK D J, SMITH B J, BRUS C P, FISHER E F, NEUBERGER J S, et al. Residential 
radon gas exposure and lung cancer: The Iowa Radon lung Cancer Study. Am J Epidemiol 2000; 151: 10911102.

18.- PINO P, OYARZÚN M, WALTER T, VON BAER D, ROMIEU I. Contaminación aérea intradomiciliaria en el área sur oriente de Santiago. Rev Med Chile 1998; 126 : 367-74.
19.- BELLO S, MICHALLAND S, SOTO M, CONTRERAS C, SALINAS J. Efectos de la exposición al humo de tabaco ambiental en no fumadores. Rev Chil Enf Respir 2005; 23: 179-92.

20.- AMERICAN ACADEMY OF PEDIATRICS. Ambient air pollution: Health Hazard to Children. Pediatrics 2004; 114: 1600-707.

Correspondencia a:

Dr. Manuel Oyarzún G.

Facultad de Medicina, Universidad de Chile.

E-mail:moyarzun@med.uchile.cl 